\title{
Massive hemoptysis related to contained rupture of syphilitic aortic aneurysm into the pulmonary parenchyma
} \author{
Turkey \\ From the Departments of ${ }^{\mathrm{a} C a r d i o v a s c u l a r}$ Surgery and ${ }^{\mathrm{b}}$ Pathology, Marmara University Faculty of Medicine, \\ Istanbul, Turkey. \\ Disclosures: Authors have nothing to disclose with regard to commercial support \\ Received for publication Jan 17, 2017; revisions received March 10, 2017; accepted for publication March 31, \\ 2017; available ahead of print May 2, 2017. \\ Address for reprints: Koray Ak, MD, PhD, Marmara Universitesi Pendik Egitim ve Arastırma Hastanesi, Ustkay- \\ narca Pendik, Istanbul, Turkey (E-mail: korayakmd@gmail.com). \\ J Thorac Cardiovasc Surg 2017;154:e23-5 \\ $0022-5223 / \$ 36.00$ \\ Copyright (C) 2017 by The American Association for Thoracic Surgery \\ http://dx.doi.org/10.1016/j.jtcvs.2017.03.134
}

Selim İsbir, MD, ${ }^{\mathrm{a}}$ Anar Hamidov, MD, ${ }^{\mathrm{a}}$ İpek Erbarut Seven, $\mathrm{MD},{ }^{\mathrm{b}}$ and Koray Ak, MD, PhD, ${ }^{\mathrm{a}}$ Istanbul,

\section{Video clip is available online.}

A brief summary about the history and management of our patient is given in Video 1.

\section{CLINICAL SUMMARY}

A 68-year-old man came to the emergency department with massive hemoptysis and sudden-onset sharp, stabbing chest pain radiating to his back. His medical history was consistent with diabetes mellitus and chronic obstructive lung disease. His initial vital signs showed a blood pressure of $85 / 50 \mathrm{~mm} \mathrm{Hg}$, a pulse of 110 beats/min, and a respiratory rate 28 breaths/min. Results of ophthalmologic examination were significant for medial deviation of the left eye. The arterial blood gas analysis was consistent with hypoxemic, hypercapnic, and respiratory acidosis. In view of the progressively worsening hypoxemia, deteriorating hemodynamics, and ongoing hemoptysis, the patient was intubated for impending respiratory failure. Massive hemoptysis did not continue after intubation. Computed tomography revealed that the ascending aorta was ruptured into the right upper lobe, which was contained by the pulmonary parenchyma (Figure $1, A$ ). The ascending aorta and root were found to be dilated (up to $60 \mathrm{~mm}$ ). There were multiple saccular protrusions from the aneurysm (Figure 1,B). The patient was rushed into the operating room.

On transesophageal echocardiography, moderately severe aortic valve insufficiency was seen. The leaflets were structurally normal. After femoral artery and vein cannulation, cardiopulmonary bypass was instituted. The patient was cooled to $20^{\circ} \mathrm{C}$, and a median sternotomy was performed during cooling. On inspection, a thick, fibrotic connection between the ascending aorta and the right pleura

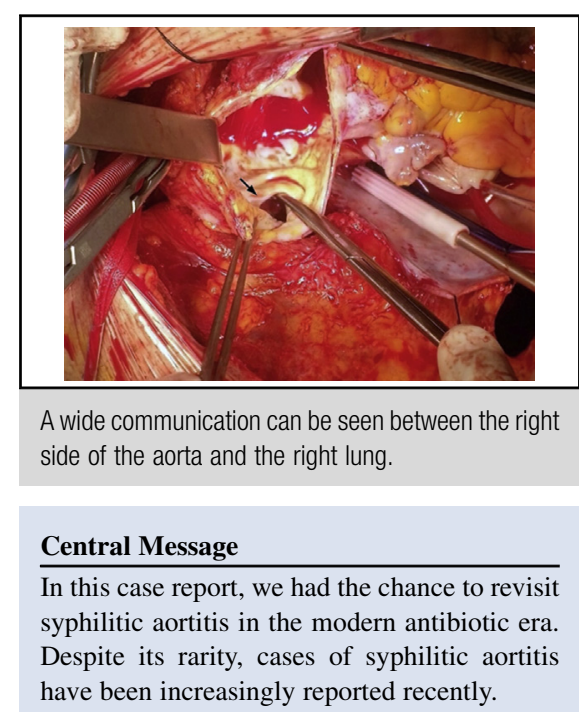

See Editorial Commentary page e27.

at the region of the pericardial reflection on the aorta was noted. There were 2 saccular protrusions coming off the aneurysm. The wall of the aneurysm was thick and heavily fibrotic. There was a chronic type contained rupture and fistulization of the aneurysm into the upper lobe of the right lung (Figure 1,C). First, the root was replaced during cooling in a Bentall procedure with a size 23 bileaflet mechanical prosthesis (Sorin, Saluggia, Italy) and a size 28 polyester graft (Intervascular; Datascope, Ciotat, France). Then, the brachiocephalic artery was cannulated for antegrade cerebral perfusion. The hemiarch replacement

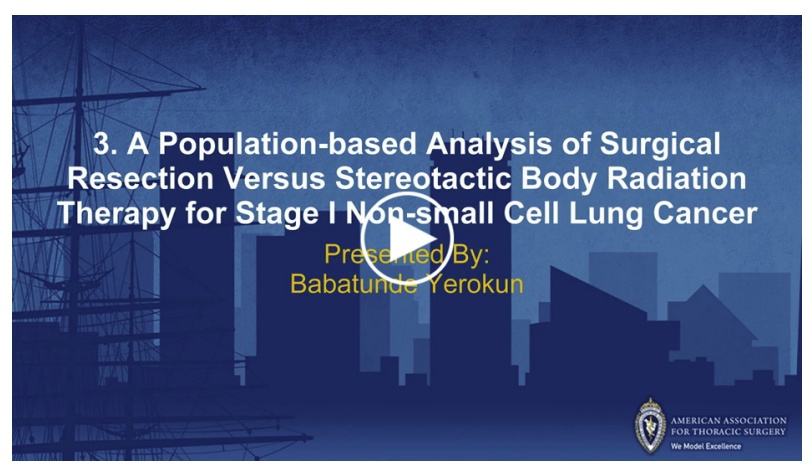

VIDEO 1. Selim İsbir summarizes the history and management of this patient with syphilitic aortitis. Video available at: http://www.jtcvsonline. org/article/S0022-5223(17)30660-8/addons. 

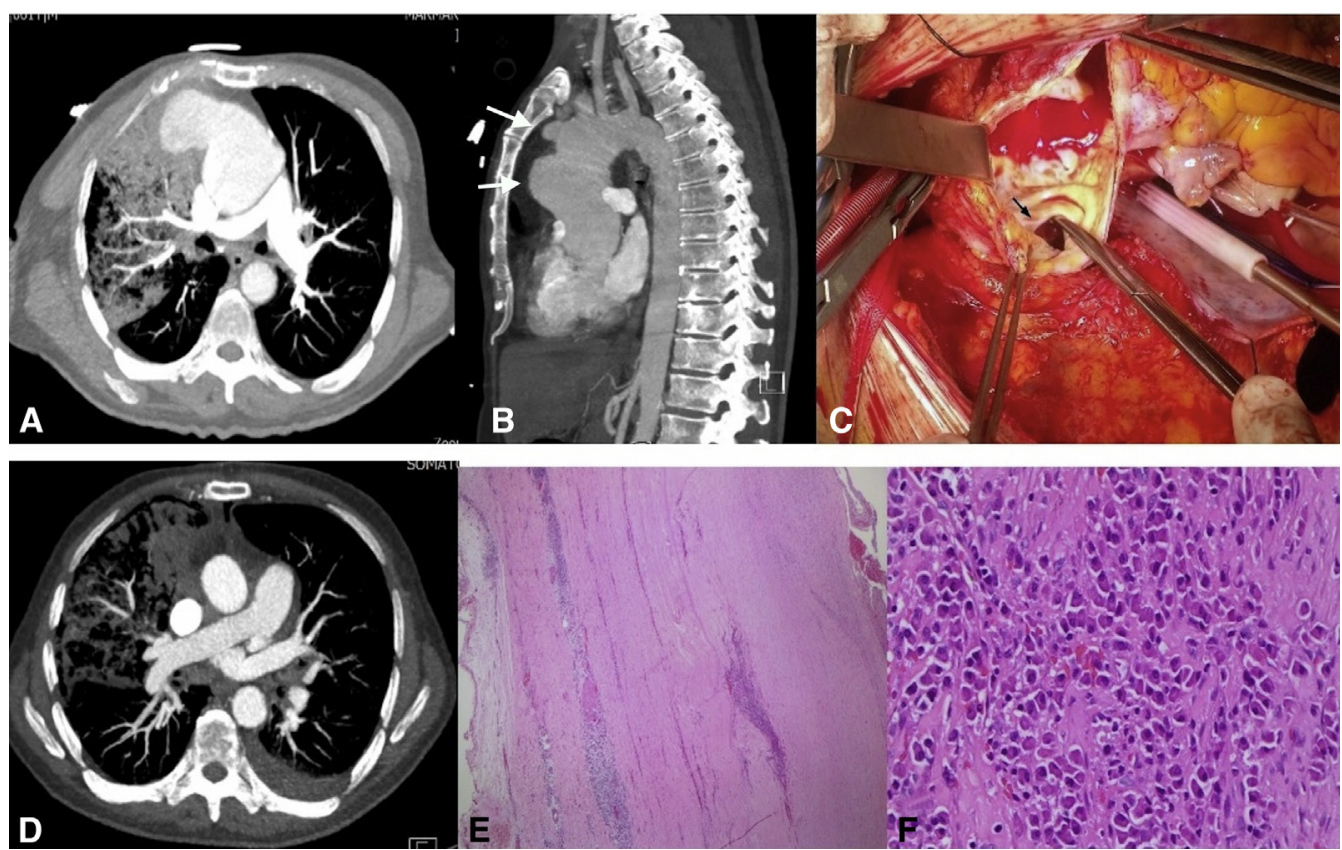

FIGURE 1. A, Computed tomographic angiography showing the contained rupture of the aorta and consolidation around the ruptured cavity in the right lung. B, Saccular protrusions from the ascending aortic aneurysm (white arrows). C, Intraoperative view from the surgeon's side. The ascending aorta has been opened. A wide communication between the right side of the aorta and the right lung can be seen (black arrow). D, Postoperative control computed tomographic angiography. E, Histologic preparation of the aneurysm wall showing chronic inflammation in the media and around the vasa vasorum (hematoxylin-eosin, original magnification $\times 10$ ). F, Histologic preparation of the aneurysm wall showing plasma cell-dominant infiltration (hematoxylin-eosin, original magnification $\times 40$ ).

was performed in an open anastomotic fashion under antegrade cerebral perfusion.

The early postoperative course was uneventful. Pathologic examination of the aneurysmal aortic tissue revealed destruction of the elastic layer and smooth muscle fibers with plasma cell-rich chronic inflammation in the tunica media and around the vasa vasorum (Figure 1, $E$ and $F$ ). Morphologic findings were considered to be suggestive of syphilitic aortitis. Postoperative laboratory analysis revealed that the patient's serum was positive for both Treponema pallidum antibody (by the T pallidum enzymelinked immunosorbent assay method) and Venereal Disease Research Laboratory Rapid Plasma Reagent (titer 1/32). A diagnosis of tertiary syphilis complicated by ascending aortic aneurysm, aortopulmonary fistula, and possible extraocular nerve palsy was made. Benzathine penicillin was prescribed for a period of 21 days after diagnosis. Three months after discharge, the patient is still doing well, with no further aneurysm formation in the remainder of the aorta seen on computed tomographic aortography.

\section{DISCUSSION}

Syphilitic aortitis occurs in the tertiary stage of the disease, 10 to 30 years after the primary infection, and is known to include ascending aortitis, ascending aorta aneurysm, aortic valvular insufficiency, and coronary stenoses. Morphologically, syphilitic aortitis causes a fusiform aneurysm of the ascending aorta in most affected patients, and superimposition of a saccular aneurysm on a fusiform aneurysm is regarded to be diagnostic of syphilitic aortitis. Obliterative endarteritis of the vasa vasorum initiates ischemic weakening of the medial layer, which further paves the way for the development of degenerative alterations in the aorta.

In patients with syphilitic aortic aneurysms, the prognosis is extremely dismal without surgical treatment, with a 2-year mortality of almost $80 \%$. Moreover, because of the saccular nature of the aneurysm and higher inflammatory activity in the aneurysmal wall, higher rates of rupture, erosion, and fistulization with such surrounding structures as the sternum, superior vena cava, and pulmonary artery than are seen in atherosclerotic aneurysms have been reported. ${ }^{2}$ In the case presented here, a chronic, contained rupture of the ascending aortic aneurysm into the right lung with related fistulization into the right bronchial tree was reported. In the literature, chronic, contained rupture of a syphilitic right subclavian artery aneurysm into the lung parenchyma and bronchial tree has been reported in 2 patients ( 1 as a postmortem case). ${ }^{3,4}$ Penicillin is prescribed for a period of 21 days in patients with the diagnosis of tertiary syphilis.

The presence of concurrent arch and descending thoracic aorta aneurysms was reported in $50 \%$ and $43 \%$, respectively, of patients with syphilitic ascending aortic 


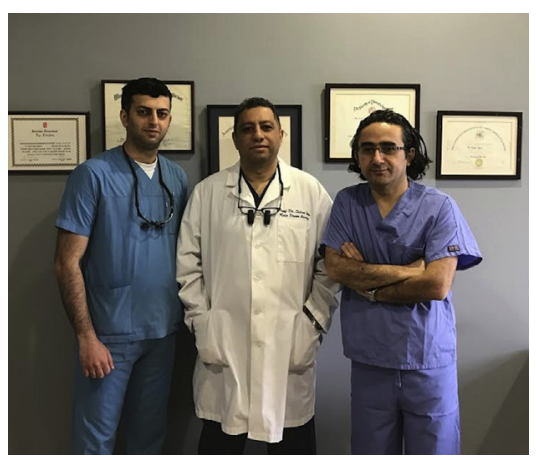

FIGURE 2. Left to right: Anar Hamidov, MD, Selim İsbir, MD, and Koray $\mathrm{Ak}, \mathrm{MD}, \mathrm{PhD}$.

aneurysms. ${ }^{1}$ Lifelong surveillance of the remainder of the aorta with serial imaging is therefore essential after surgery.
In conclusion, despite its rarity, atypical clinical presentation (such as hemoptysis, cardiac tamponade, and superior vena cava syndrome), thicker aneurysmal wall, and a pattern of saccular on fusiform aneurysm pattern should raise suspicion of syphilitic ascending aortic aneurysm. The surgical team is shown in Figure 2.

\section{References}

1. Roberts WC, Barbin CM, Weissenborn MR, Ko JM, Henry AC. Syphilis as a cause of thoracic aortic aneurysm. Am J Cardiol. 2015;116:1298-303.

2. Maleszewski JJ. Inflammatory ascending aortic disease: perspectives from pathology. J Thorac Cardiovasc Surg. 2015;149(2 Suppl):S176-83.

3. Sopher IM, Spitz WU. Exsanguinating hemoptysis due to a ruptured syphilitic aneurysm. J Forensic Med. 1971;18:118-21.

4. Boundy K, Bignold LP. Syphilitic aneurysm of the right subclavian artery presenting with hemoptysis. Aust N Z J Med. 1987;17:533-5.

5. Ghanem KG. Management of adult syphilis: key questions to inform the 2015 Centers for Disease Control and Prevention Sexually Transmitted Diseases Treatment Guidelines. Clin Infect Dis. 2015;61(Suppl 8):S818-36. 